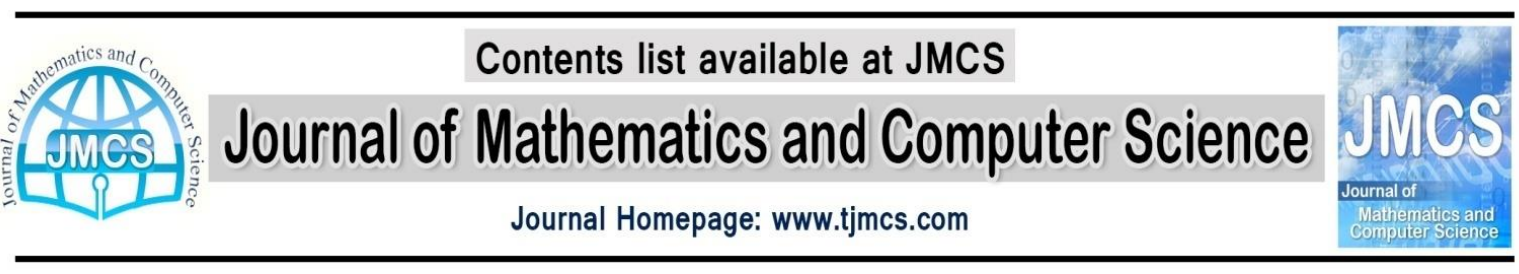

\title{
Best Approximation by Upward Sets
}

\author{
Zeinab Soltani ${ }^{1, *}$, Hamid Reza Goudarzi ${ }^{1,+}$ \\ ${ }^{1}$ Department of Mathematics, Faculty of Scinence, Yasouj University, Yasouj, Iran. \\ *zeinabsoltani62@yahoo.com \\ +goudarzi@mail.yu.ac.ir
}

\begin{abstract}
Article history:
Received November 2014

Accepted January 2015

Available online January 2015
\end{abstract}

\begin{abstract}
In this paper we prove some results on upward subsets of a Banach lattice $X$ with a strong unit. Also we study the best approximation in $X$ by elements of upward sets, and we give the necessary and sufficient conditions for any element of best approximation, by a closed subset of $X$.
\end{abstract}

Keywords: Banach lattice, Best approximation, Proximinal set, Upward set.

\section{Introduction}

The theory of best approximations is an important subject in Functional Analysis. It is a very extensive field which has various applications ([2], [5], [7] and [10] ). Also the problems of best approximation by elements of convex sets are welldeveloped and have many applications in different areas of Mathematics ( [3], [4], [6], [8] and [12]). Downward and upward sets are not necessarily convex and since, convexity is sometimes a very restrictive assumption, so we can use the so-called downward and upward sets as good tools in the study of best approximation by closed and not necessarily convex sets. Best approximation by downward sets and their properties have been studied by several authors (for example see [1]).

The approximation properties of upward sets play a crucial role in this paper. We study some aspects of best approximation by elements of closed upward sets, in a Banach lattice $X$ with a strong unit. We show that a closed upward set is proximinal, and we derive the necessary and sufficient conditions for the uniqueness of best approximation. As we reminded, an upward set is not necessarily convex. We show that this set is abstract convex with respect to a certain set of elementary functions (for the definition of abstract convexity, see [11]). This fact allows us to examine the separation properties of upward sets and gives the necessary and sufficient conditions for best approximations. 


\section{Preliminaries}

In this section, we introduce some basic definitions for the next sections. Let $G$ be a non empty subset of a normed linear space $X$. An element $g_{0} \in G$ is called a best approximation to $x \in X$ from $G$ if for every $g \in G$,

$$
\left\|x-g_{0}\right\| \leq\|x-g\| .
$$

On the other hand, recall that (see e.g. [13]) a point $g_{0} \in G$ is called a best approximation to $x \in X$ if

$$
\left\|x-g_{0}\right\|=d(x, G),
$$

Where

$$
d(x, G)=\inf _{g \in G}\|x-g\| .
$$

The set of all such elements $g_{0} \in G$ (called best approximations to $\left.x \in X\right)$ is denoted by $P_{G}(\mathrm{x})$. Thus

$$
P_{G}(\mathrm{x})=\left\{g_{0} \in \mathrm{G}: \quad\left\|\mathrm{x}-g_{0}\right\|=\mathrm{d}(\mathrm{x}, \mathrm{G})\right\} .
$$

Hence $P_{G}$ defines a mapping from $X$ into the power set of $G$, called metric projection onto $G$ ( other names are nearest point mapping and proximity map). If $P_{G}(x)$ contains at least one element, then the subset $G$ is call a proximinal set. In other words, if $P_{G}(x)=\varnothing$, then $G$ is called a proximinal set. Also if each element $x \in X$ has a unique best approximation in $G$, then $G$ is called a Chebyshev subset of $X$.

It is well-known that $P_{G}(x)$ is a closed and bounded subset of $X$. If $x \in G$ then. $P_{G}(x)$ is located in the boundary of $G$.

Definition 2.1 ( [14]) A vector space $X$ which is ordered by a relation $\leq$, is called a vector lattice if any two elements $x, y \in X$ have a least upper bound denoted by $x \vee y=\sup (x, y)$, a greatest lower bound denoted by $x \wedge y=\inf (x, y)$ and the following properties are satisfied:

(1) $x \leq y$ implies that $x+z \leq y+z$, for all $x, y, z \in X$,

(2) $x \geq o$ implies that $t x \geq 0$, for all $x \in X$ and $t \in R^{+}$.

If a vector lattice $X$ is equipped with a norm $\|$.$\| for which$

(3) $|x| \leq|y|$ implies $\|x\| \leq\|y\|$, for $x, y \in X$,

then $X$ (equipped with $\leq$ and $\|$.$\| ) is called a normed vector lattice. A complete normed vector$ lattice is called a Banach lattice.

Example 2.2 Let $S$ be a set, equipped with a sigma-field $\sigma$. The space $L^{\infty}(S, \sigma)$ of all bounded, $\sigma$-measurable real functions on $S$ is a vector lattice for the pointwise ordering $f \leq g$ means $f(s) \leq g(s), \forall s \in S$. The supremum norm, defined by $\|f\|=\sup _{s \in S}|f(s)|$, satisfies in 2.1(3) and the space $L^{\infty}(S, \sigma)$ is a normed vector lattice. The space $L^{\infty}(S, \sigma)$ is also complete by supremum norm, in the sense that Cauchy sequences converge to bounded, measurable limit functions. 
If $X$ is a vector lattice, an element $\mathbf{1} \in X$ is called a strong unit if for each $x \in X$, there exists $0<$ $\alpha \in \mathrm{R}$ such that $x \leq \alpha \mathbf{1}$.

Using a strong unit $\mathbf{1}$, we can define a norm on $X$ by

$$
\|x\|=\inf \{\alpha>0:|x| \leq \alpha \mathbf{1}\} \quad \forall x \in X
$$

Then

$$
B(x, r):=\{y \in X:\|x-y\| \leq r\}=\{y \in X: x-r \mathbf{1} \leq y \leq x+r \mathbf{1}\}
$$

Also we have

$$
|x| \leq\|x\| \mathbf{1} \quad \text { for all } \quad x \in X .
$$

$l^{\infty}\left(\mathrm{S}, \sum, \mu\right)$ denotes the lattice of all essentially bounded functions defined on the space $S$ with a $\sigma-$ algebra of measurable sets, $\Sigma$, and a measure $\mu$. Also th lattice of all bounded functions defined on $X$ is an example of Banach lattices with the strong units.

Recall that a subset $G$ of an ordered set $X$ is said to be upward if

$$
g \in G, g \leq x \Rightarrow x \in G \text {. }
$$

If $X$ is a normed linear space and $\mathrm{G}$ is a subset of $X$, we shall denote by $\operatorname{int} G, c l G$ and $b d G$ the interior, the closure and the boundary of $G$, respectively. If $X$ is a lattice and there exists the greatest elements of $G$, we shall denote it by $\max G$.

\section{Upward sets and their approximation properties}

Let $X$ be a Banach lattice with a strong unit $\mathbf{1}$. In this section we investigate the best approximations in $X$ by elements of upward sets. In particular, we show that the greatest element, in the set of best approximations, exists.

Proposition 3.1 Let $G$ be an upward subset of $X$ and $x \in X$. Then the following statements are true:

(1) If $x \in G$, then $x+\varepsilon \mathbf{1} \in$ int $G, \forall \varepsilon>0$,

(2) $\operatorname{int} G=\{x \in X: x-\varepsilon \mathbf{1} \in G$, for some $\varepsilon>0\}$.

Proof: (1) Let $\varepsilon>0$ be given and $x \in G$. suppose that

$$
W=\{y \in X:\|y-(x+\varepsilon \mathbf{1})\|<\varepsilon\},
$$

be an open neighborhood of $(x+\varepsilon \mathbf{1})$. Then, by (3),

$$
W=\{y \in X: x<y<x+2 \varepsilon \mathbf{1}\} .
$$

Since $G$ is an upward set and $x \in G$, it follows that $W \subset G$, and so $x+\varepsilon \mathbf{1} \in \operatorname{int} G$. 
(2) Let $x \in \operatorname{int} G$. Then there exists $\varepsilon_{0}>0$ such that the closed ball $B\left(x, \varepsilon_{0}\right) \subset G$. In view of (3), $x-\varepsilon_{0} \mathbf{1} \in G$. Conversely, suppose that there exists $\varepsilon>0$ such that $x-\varepsilon \mathbf{1} \in G$. Then, as we saw, $x=(x-\varepsilon \mathbf{1})+\varepsilon \mathbf{1} \in$ int $G$, which completes the proof.

Corollary 3.2 Let $\mathrm{G}$ be a closed upward subset of $\mathrm{X}$ and $g \in G$. Then $g \in b d G$ if and only if $g-$ $\alpha \mathbf{1} \notin G$, for each $\alpha>0$.

Lemma 3.3 Let $G$ be a closed upward subset of $X$. Then $G$ is proximinal in $X$.

Proof: Let $x_{0} \in X \backslash G$ be arbitrary and $r:=d\left(x_{0}, G\right)=$ inf $g \in G\left\|x_{0}-g\right\|>0$. This implies that for each $\varepsilon>0$, there exists $g_{\varepsilon} \in G$ such that $\left\|x_{0}-g_{\varepsilon}\right\|<r+\varepsilon$. Then, by (3), we have

$$
-(r+\varepsilon) \mathbf{1} \leq g_{\varepsilon}-x_{0} \leq(r+\varepsilon) \mathbf{1} \text {. }
$$

Let $g_{0}=x_{0}+\mathrm{r} \mathbf{1}$. Then, we have

$$
\left\|x_{0}-g_{0}\right\|=\|-r \mathbf{1}\|=r=d\left(x_{0}, G\right),
$$

and hence $g_{0}+\varepsilon \mathbf{1}=x_{0}+r \mathbf{1}+\varepsilon \mathbf{1}=x_{0}+(r+\varepsilon) \mathbf{1} \geq g_{\varepsilon}$. Since G is upward and $g_{\varepsilon} \in$ $G$, it follows that $g_{0}+\varepsilon \mathbf{1} \in G$, for each $\varepsilon>0$. Since $\mathrm{G}$ is closed, we have $g_{0} \in \mathrm{G}$ and then $g_{0} \in$ $P_{G}\left(x_{0}\right)$. Thus the result follows.

Remark 3.4 We proved that for each $x_{0} \in X \backslash G$, the set $P_{G}\left(x_{0}\right)$ contain the element $g_{0}=x 0+$ $r \mathbf{1}$ with $r=d\left(x_{0}, G\right)$. If $x_{0} \in G$ then $g_{0}=x_{0}$ and $P_{G}\left(x_{0}\right)=\left\{g_{0}\right\}$.

Proposition 3.5 Let $G$ be a closed upward subset of $X$ and $x_{0} \in X$. Then there exists the greatest element $g_{0}=\max P_{G}\left(x_{0}\right)$ of the set $P_{G}\left(x_{0}\right)$, namely $g_{0}=x_{0}+r \mathbf{1}$, where $r:=d\left(x_{0}, G\right)$.

Proof: If $x_{0} \in G$, then the result holds. Assume that $x_{0} \notin G$ and $g_{0}=x_{0}+r \mathbf{1}$.

Then, by remark 3.4, we have $g_{0} \in P_{G}\left(x_{0}\right)$. By applying (3) and the equality $\left\|x_{0}-g_{0}\right\|=r$, we get

$$
x \leq x_{0}+r \mathbf{1}=g_{0} \quad \text { for all } \quad x \in B\left(x_{0}, r\right) .
$$

This implies that $g_{0}$ is the greatest element of the closed ball $B\left(x_{0}, r\right)$. Now, let $g \in P_{G}\left(x_{0}\right)$ be arbitrary. Then $\left\|x_{0}-g\right\|=r$, and so $g \in B\left(x_{0}, r\right)$. Therefore $g \leq g_{0}$ and hence, $g_{0}$ is the greatest element of $P_{G}\left(x_{0}\right)$.

Corollary 3.6 Let $G$ be a closed upward subset of $X, x_{0} \in X$ and $g_{0}=\max P_{G}\left(x_{0}\right)$. Then, $g_{0} \geq x_{0}$.

Corollary 3.7 Let $G$ be a closed upward subset of $X$ and $x \in X$ be arbitrary. Then

$$
d(x, G)=\min \{\alpha \geq 0: x+\alpha \mathbf{1} \in G\} .
$$

Proof : Let $A=\{\alpha \geq 0: x+\alpha \mathbf{1} \in G\}$. If $x \in G$ then $x+0 \mathbf{1}=x \in G$, and so $\min A=$ $0=d(x, G)$. Suppose that $x \notin G$ then $r:=d(x, G)>0$. Let $\alpha>0$ be arbitrary such that $x+$ $\alpha \mathbf{1} \in G$. Thus, we have

$$
\alpha=\|\alpha \mathbf{1}\|=\|(x+\alpha \mathbf{1})-x\| \geq d(x, G)=r .
$$


Since, by proposition 3.5, $x+r \mathbf{1} \in G$, it follows that $r \in A$. Hence, $\min A=r$ which completes the proof. $\square$

\section{Characterization of best approximation by upward sets}

In this section, we present the characterization of upward sets in terms of separation from outside points. Throughout this section, $\mathrm{X}$ is a Banach lattice. Let $\varphi: X \times X \rightarrow R$ be a function defined by

$$
\varphi(x, y):=\sup \{\alpha \in R: \alpha \mathbf{1} \leq x+y\}, \quad(x, y \in X) .
$$

Since $\mathbf{1}$ is a strong unit, it follows that the set $\{\alpha \in R: \alpha \mathbf{1} \leq x+y\}$ is non empty and bounded from above ( by the number $\|x+y\|$ ). Clearly this set is closed. It follows from the aforesaid and the definition of $\varphi$ that the function $\varphi$ enjoys the following properties.

$$
\begin{array}{ccc}
-\infty<\varphi(x, y) \leq\|x+y\|, & \text { for all } & x, y \in X ; \\
\varphi(x, y) \mathbf{1} \leq x+y, & \text { for all } & x, y \in X ; \\
\varphi(x, y)=\varphi(y, x), & \text { for all } & x, y \in X ; \\
\varphi(x,-x)=\sup \{\alpha \in R: \alpha \mathbf{1} \leq x-x=0\}=0, \text { for all } \quad x \in X ; \\
\varphi(x, y+\alpha \mathbf{1})=\varphi(x, y)+\alpha, \quad \text { for all } & x, y \in X \text { and } \alpha \in R ; \\
\varphi(x+\alpha \mathbf{1}, y)=\varphi(x, y)+\alpha & \text { for all } & x, y \in X \text { and } \alpha \in R ; \\
\varphi(\gamma x, \gamma y)=\gamma \varphi(x, y), & \text { for all } & x, y \in X \text { and } \gamma>0 .
\end{array}
$$

For each $y \in X$, define the function $\varphi_{y}: X \rightarrow R$ by

$$
\varphi_{y}(x):=\varphi(x, y) \quad \text { for all } \quad x \in X .
$$

The function $f: X \rightarrow R$ is called topical if this function is increasing $(x \geq y \Rightarrow(x) \geq f(y))$ and plus-homogeneous $(f(x+\lambda \mathbf{1})=f(x)+\lambda$ for all $x \in \mathrm{X}$ and $\lambda \in \mathrm{R})$. The definition of topical function in finite dimensional case can be found in [11].

Lemma 4.1 The function $\varphi_{y}$ defined by (13) is topical.

Proof: we try to check the conditions. Let $x, z \in X$ with $x \leq z$. Then $\{\alpha \in R: \alpha \mathbf{1} \leq x+y\} \subset$ $\{\alpha \in R: \alpha \mathbf{1} \leq z+y\}$. Hence,

$$
\varphi_{y}(x)=\sup \{\alpha \in R: \alpha \mathbf{1} \leq x+y\} \leq \sup \{\alpha \in R: \alpha \mathbf{1} \leq z+y\}=\varphi_{y}(z) .
$$

Let $x \in X$ and $\lambda \in R$ be arbitrary. Then

$$
\begin{aligned}
\varphi_{y}(x+\lambda \mathbf{1}) & =\sup \{\alpha \in R: \alpha \mathbf{1} \leq x+\lambda \mathbf{1}+y\} \\
& =\sup \{\alpha \in R:(\alpha-\lambda) \mathbf{1} \leq x+y\} \\
& =\sup \{\beta+\lambda \in R: \beta \mathbf{1} \leq x+y\} \\
& =\sup \{\beta \in R: \beta \mathbf{1} \leq x+y\}+\lambda=\varphi_{y}(x)+\lambda .
\end{aligned}
$$


Proposition 4.2 The function $\varphi_{y}$ is Lipschitz continuous.

Proof: Let $x, z \in X$ be arbitrary. Since $|x-z| \leq\|x-z\| \mathbf{1}$, it follows that

$$
z-\|x-z\| \mathbf{1} \leq x \leq z+\|x-z\| \mathbf{1} \text {. }
$$

In view of lemma 4.1, we have

$$
\varphi_{y}(z)-\|x-z\| \leq \varphi_{y}(x) \leq \varphi_{y}(z)+\|x-z\|,
$$

and hence

$$
\varphi_{y}(x)-\varphi_{y}(z) \mid \leq\|x-z\| .
$$

Therefore, $\varphi_{y}$ is Lipschitz continuous.

As a direct result of (14), we have:

Corollary 4.3 The function $\varphi$ defined by (5) is continuous.

Lemma 4.4 Let $G$ be a closed upward subset of $X, y_{0} \in b d G$ and let $\varphi$ be the function defined by (5). Then, $\varphi\left(-g, y_{0}\right) \leq 0$, for all $g \in G$.

Proof: Assume that there exists $g_{0} \in G$ such that $\varphi\left(g_{0}, y_{0}\right)>0$. Then $\sup \{\alpha \in R: \alpha \mathbf{1} \leq$ $\left.-g_{0}+y_{0}\right\}>0$. So there exists $\alpha_{0}>0$ such that $\alpha_{0} \mathbf{1} \leq-g_{0}+y_{0}$. This means that $\alpha_{0} \mathbf{1}-$ $y_{0} \leq-g_{0}$. Therefore $y_{0}-\alpha_{0} \mathbf{1} \geq g_{0}$. Since $G$ is upward and $g_{0} \in G$, it follows that $y_{0}-$ $\alpha_{0} \mathbf{1} \in G$. So, by proposition 3.1 (2), we have $y_{0} \in \operatorname{int} G$. This is a contradiction, which completes the proof.

Now, we give the characterization of upward sets in terms of separation from outside points. For an easy reference we present the following theorem.

Theorem 4.5 Let $G$ be a subset of $X$ and $\varphi$ be the coupling function of (5). Then the following statements are equivalent:

(1) $G$ is an upward set,

(2) For each $x \in X \backslash G$, we have

$$
\varphi(-g, x)<0, \forall g \in G,
$$

(3) For each $x \in X \backslash G$, there exists $l \in X$ such that

$$
\varphi(-g, l)<0 \leq \varphi(-x, l) \quad \forall g \in G .
$$

Proof:(1) $\Rightarrow$ (2). Suppose that $G$ is an upward set and that exists $x \in X \backslash G, g \in G$ such that $\varphi(-g, x) \geq 0$. Then, by (7) we have $0 \leq \varphi(-g, x) \mathbf{1} \leq x-g$, and so $x \geq g$. Since $\mathrm{G}$ is an upward set and $g \in G$, it follows that $x \in G$. This is a contradiction.

(2) $\Rightarrow$ (3). Assume that (2) holds and $x \in X \backslash G$ is arbitrary. Then by hypothesis, we have

$$
\varphi(-g, x)<0 \quad \forall g \in G .
$$


Now, let $l=x \in X$, using (9), for each $g \in G$, we have :

$$
\varphi(-g, l)=\varphi(-g, x)<0=\varphi(-x, x)=\varphi(-x, l) .
$$

(3) $\Rightarrow$ (1). Suppose that (3) holds and $G$ is not an upward set. Then there exists $g_{0} \in G$ and $x_{0} \in$ $X \backslash \mathrm{G}$ with $x_{0} \geq g_{0}$. It follows, by hypothesis, that there exists $l \in X$ such that

$$
\varphi(-g, l)<0 \leq \varphi\left(-x_{0}, l\right), \quad \forall g \in G .
$$

Since $\varphi(., l)$ is increasing, we have

$$
0 \leq \varphi\left(-x_{0}, l\right) \leq \varphi\left(-g_{0}, l\right)
$$

This contradicts (15) .

Theorem 4.6 Let $\varphi$ be the function defined by (5). Then for a subset $G$ of $X$ the following statements are equivalent:

(1) $G$ is a closed upward subset of $X$,

(2) $G$ is upward, and for each $x \in X$ the set

$$
H=\{\alpha \in R: x-\alpha \mathbf{1} \in G\}
$$

is closed in $R$,

(3) For each $x \in X \backslash G$, there exists $l \in X$ such that

$$
\varphi(-g, l)<0<\varphi(-x, l), \quad(g \in G)
$$

(4) For each $x \in X \backslash G$, there exists $l \in X$ such that

$$
\sup _{g \in G} \varphi(-g, l)<\varphi(-x, l) \text {. }
$$

Proof: $(1) \Rightarrow(2)$. Assume that $G$ is a closed upward subset of $X$ and let $x \in X, \alpha_{k} \in R, x-\alpha_{k} \mathbf{1} \in$ $G(k=1,2, \ldots)$ and $\alpha_{k} \rightarrow \alpha \in \mathrm{R}$. Then, we have

$$
\|\left(x-\alpha_{k} \mathbf{1}\right)-\left(x-\alpha \mathbf{1}\|=\|\left(\alpha-\alpha_{k}\right) \mathbf{1} \|=\left|\alpha-\alpha_{k}\right| \rightarrow 0 \text {, as } k \rightarrow+\infty .\right.
$$

Since $x-\alpha_{k} \mathbf{1} \in G,(k=1,2, \ldots)$, and $G$ is closed, it follows that $x-\alpha \mathbf{1} \in G$, and so $\alpha \in H$. Hence, $H$ is a closed subset of $R$.

(2) $\Rightarrow$ (3). Suppose that (2) holds and $x \in X \backslash G$ is arbitrary. We claim that there exists $\alpha_{0}>0$ such that $-\alpha_{0} \in H$. Indeed, if $-\alpha \in H$, for all $\alpha>0$, then due to the closedness of $\mathrm{H}$, we have $0 \in$ $H$. This implies that $x=x-0 \mathbf{1} \in G$. This is a contradiction. Now, let $l=x+\alpha_{0} \mathbf{1} \in X$. We show that $\varphi(-g, l)<0$, for all $g \in G$. Assume that there exists $g_{0} \in G$ such that $\varphi\left(-g_{0}, l\right) \geq 0$. Then by (7) we have $0 \leq \varphi\left(-g_{0}, l\right) \mathbf{1} \leq-g_{0}+l$, and so $g_{0} \leq l=x+\alpha_{0} \mathbf{1}$. Since $G$ is an upward set and $g_{0} \in G$, it follows that $x+\alpha_{0} \mathbf{1} \in G$, and consequently $-\alpha_{0} \in H$. This is a contradiction. Hence, $\varphi(-g, l)<0$, for all $g \in G$. On the other hand, we have

$$
\begin{aligned}
\varphi(-x, l) & =\sup \{\alpha \in R: \alpha \mathbf{1} \leq-x+l\} \\
& =\sup \left\{\alpha \in R: \alpha \mathbf{1} \leq-x+\alpha_{0} \mathbf{1}+x=\alpha_{0} \mathbf{1}\right\}
\end{aligned}
$$




$$
\begin{aligned}
& =\sup \left\{\alpha \in R:\left(\alpha-\alpha_{0}\right) \mathbf{1} \leq 0\right\} \\
& =\sup \left\{\lambda+\alpha_{0} \in R ; \lambda \mathbf{1} \leq 0\right\} \\
& =\sup \{\lambda \in R: \lambda \mathbf{1} \leq 0\}+\alpha_{0}=\alpha_{0}>0 .
\end{aligned}
$$

(3) $\Rightarrow(4)$ is abvious.

(4) $\Rightarrow(1)$. Suppose that (4) holds and that $G$ is not an upward set. Then there exists $g_{0} \in G$ and $x_{0} \in X \backslash G$ with $x_{0} \geq g_{0}$. By hypothesis, there exists $l \in X$ such that

$$
\sup _{g \in G} \varphi(-g, l)<\varphi\left(-x_{0}, l\right) \text {. }
$$

Since $\varphi(., l)$ is increasing, it followes that

$$
\varphi\left(-x_{0}, l\right) \leq \varphi\left(-g_{0}, l\right) \leq \sup _{g \in G} \varphi(-g, l)<\varphi\left(-x_{0}, l\right)
$$

This is a contradiction. Hence, $G$ is an upward set. Finally, assume that $G$ is not closed. Then there exists a sequence $\left\{g_{n}\right\}_{n \geq 0} \subset G$ and $x_{0} \in X \backslash G$ such that $\left\|g_{n}-x_{0}\right\| \rightarrow 0$, as $n \rightarrow$ $+\infty$. Since $x_{0} \in X \backslash G$, by hypothesis, there exists $l \in X$ such that

$$
\sup _{g \in G} \varphi(-g, l)<\varphi\left(-x_{0}, l\right) .
$$

Thus, we have

$$
\varphi\left(-g_{n}, l\right) \leq \sup _{g \in G} \varphi(-g, l), \quad \forall n \geq 1 .
$$

By continuity of $\varphi_{l}=\varphi(., l)$, it follows that $\varphi\left(-x_{0}, l\right) \leq \sup _{g \in G} \varphi(-g, l)$. This is a contradiction, which completes the proof.

Lemma 4.7 Let $G$ be a closed upward subset of $X, g_{0} \in b d G$ and $l=g_{0}$. Let $\varphi$ be defined by (5). Then

$$
\varphi(g, l) \leq 0=\varphi\left(-g_{0}, l\right), \quad \forall g \in G .
$$

Proof: Since $g_{0} \in b d G$, it follows, by lemma 4.4, that

$$
\varphi(g, l)=\varphi\left(g, g_{0}\right) \leq 0, \quad \forall g \in G .
$$

Also, we have

$$
\begin{aligned}
\varphi\left(-g_{0}, l\right) & =\sup \left\{\alpha \in R: \alpha \mathbf{1} \leq-g_{0}+l\right\} \\
& =\sup \left\{\alpha \in R: \alpha \mathbf{1} \leq-g_{0}+g_{0}=0\right\} \\
& =0
\end{aligned}
$$

\section{References}

[1] H. Alizadeh Nazarkandi, "Downward sets and their topological propertices", World Applied Sciences journal. 18(11) (2012), 1630 - 1634.

[2] H. Asnashari, “Two continvity concepts in Approximation Theory”, J. Tjmcs, 4 (2012), 32-36. 
[3] C. K. Chui, F. Deutsch, J. D. Ward, "Constrained best approximation in Hilbert spaces", Conster. Approx., 6 (1990), 35-64.

[4] C. K. Chui, F. Deutsch, J. D. Ward, "Constrained best approximation in Hilbert spaces II', J. Approx. Theory, 71 (1992), 213-238.

[5] F. Deutsch, "Best approximation in inner product spaces", Springer - Velage, New York, (2000).

[6] F. Deutsch, W. Li, J. D. Ward, "A dual approach to constrained in- terpolation from a convex subset of Hilbert spaces", J. Approx. Theory, 90(1997), 385-414.

[7] R. A. Halmos, "A course on Optimization and Best approximation", CLNM257, Springer, (1972).

[8] V. Jeyakumar, H. Mohebi, “Limiting and ع-subgradient characterizations of constrained best approximation", J. Approx. Theory, 135 (2005), 145-159.

[9] J. E. Martinez - Legaz, A. M. Rubinov, I. Singer, "Downward sets and their sepration and approximation properties", J. Global Optimization, 23(2002), 111-137.

[10] H. Shojaei, R. Mortezaei, "Common Fixed Point for Affine Self Maps Invariant Approximation in P-normed Spaces", J.Tjmcs, 6(2013), 201-209.

[11] I. Singer, "Abstract convex Analysis", Wiley-Interscience, New York, (1987).

[12] I. Singer, "Best approximation in normed linear spaces by elements of linear subspaces", Springer-Verlag, New York, (1970).

[13] I. Singer, "The theory of best approximation and Functional Analysis", Re- gional Conference Series in Applied Mathematics, No. 13, (1974).

[14] B. Z. Vulikh, "Introduction to the theory of partially ordered vector spaces", Wolters Noordhoff, Groningen, (1967). 